\title{
Dispositif expérimental d'analyse des performances de cellules photovoltaïques
}

\author{
Jean-Paul Bécar*, Fabrice Robert, Jean-Charles Canonne*, Laurent Vermeiren**, \\ *LAMAV FR-CNRS 2956, **LAMIH UMR CNRS 8530, \\ \{jean-paul.becar,fabrice.robert,jean-charles.canonne,laurent.vermeiren\}@univ-valenciennes.fr \\ IUT GEII, Le Mont-Houy 59313 Valenciennes Cedex,
}

\begin{abstract}
RÉSUMÉ : L'article rapporte un travail de conception, de réalisation et d'utilisation d'un dispositif expérimental d'analyse de performances de cellules photovoltaïques. Initié à son origine par des questionnements d'étudiants d'un Institut Universitaire de Technologie relatifs aux énergies nouvelles et plus particulièrement à l'énergie solaire il s'est intégré au fil du temps dans un projet pluridisciplinaire de pilotage en position d'un système autonome d'orientation de panneaux photovoltaïques. La réalisation d'un tel dispositif a nécessité des connaissances élémentaires d'astronomie, de mécanique et d'électronique. Pour la partie astronomie le choix s'est arrêté sur le modèle géocentrique de Ptolémée. Il a permis de définir des outils de mesure, de calcul et d'animation de la course du soleil dans le ciel. La partie mécanique a été confiée à d'autres étudiants spécialistes en Conception Mécanique Assistée par Ordinateur. La partie électronique, du schéma du circuit à son branchement jusqu'aux mesures, est du domaine de compétence des étudiants en Génie Electrique et Informatique Industrielle. Le travail réalisé a non seulement enrichi les connaissances des étudiants mais aussi soulevé de nombreuses idées et applications nouvelles sur cette thématique.
\end{abstract}

Mots clés : modélisation mathématique, mesures, instrumentation, pédagogie par projet, outils logiciels, mesure du temps, modèle de Ptolémée, équation du temps, énergies renouvelables.

\section{INTRODUCTION}

Ce document relate une partie achevée d'un projet pluridisciplinaire de conception, réalisation, expérimentation et automatisation d'un support autonome de panneau photovoltaïque. Les travaux présentés ici ont rassemblé des enseignants d'Informatique, de Mathématiques, de Génie Electrique et d'Automatique. Durant près de deux années universitaires, ils ont supervisé selon leur domaine de compétence, quatre étudiants d'un Institut Universitaire de Technologie de la spécialité Génie Electrique et Informatique Industrielle, et quatre étudiants spécialistes en Conception Mécanique Assistée par Ordinateur. Leur projet de fin d'études était la réalisation d'un dispositif de mesures de performances de cellules photovoltaïques [1].

Cependant, il a été préalablement nécessaire de déterminer des paramètres solaires. Le modèle géocentrique de Ptolémée a été retenu pour la facilité de compréhension et d'implémentation qu'il offrait. C'est en section 2 que les trois outils de mesures et de calcul d'éphémérides sont exposés. Le premier outil est un théodolite de fortune. Il effectue des mesures approximatives des coordonnées locales du soleil. Le second est un logiciel animant la course du soleil à une date donnée, il indique également ses coordonnées. Enfin, un algorithme de calcul établit ces coordonnées à partir du modèle géocentrique. Il est validé au travers du calcul d'éphémérides.

La section 3 décrit le dispositif expérimental de mesures de performances des cellules photovoltaïques, le protocole de mesures, la série de résultats et les conclusions expérimentales. L'appareil a la forme d'un support mécanique stable et transportable sur lequel sont branchées des cellules photovoltaïques selon le schéma série-parallèle. Grâce à ses deux degrés de libertés, il peut ainsi s'orienter dans le flux solaire et satisfaire un des objectifs des travaux entrepris : la mesure du gain énergétique d'un panneau orientable par rapport à un panneau fixe.

La section 4 tire un certain nombre de conclusions et propose des perspectives de développement et d'ouverture.

Le schéma de réalisation du support et les fonctions Maple ${ }^{\mathrm{TM}}$ de calcul des éphémérides figurent en annexe.

\section{LA MESURE ET LE CALCUL DES ÉPHÉ- MÉRIDES}

Cette section décrit la démarche scientifique mise en œuvre pour l'observation de la course du soleil, la mesure des paramètres utiles à sa modélisation, puis les applications sous Matlab ${ }^{\circledR}$ et Maple $^{\mathrm{TM}}$ et enfin la comparaison des différents résultats.

Le modèle géocentrique de Ptolémée plutôt que le modèle héliocentrique [2] a été retenu d'une part pour sa précision suffisante dans le cadre du projet et d'autre part pour sa facilité de compréhension et d'implémentation.

L'azimut et l'élévation représentent les paramètres utiles pour définir la position du Soleil. L'azimut est l'angle que fait la direction Sud avec celle du soleil. L'observateur occupe le sommet de l'angle. L'azimut est compté positivement du Sud vers l'Ouest. La hauteur ou élévation est l'angle en degrés que fait l'axe soleil-observateur avec l'horizon local.

\subsection{Le théodolite}

Il se compose d'une boîte en carton parallélépipédique oblongue (figure 1). Elle est percée d'un trou de $2 \mathrm{~mm}$ 
de diamètre au centre d'une de ses faces et munie d'une cible sur la face intérieure opposée qui reçoit la tache lumineuse évitant ainsi l'observation directe du soleil. La face latérale est munie d'un quadrant de résolution $1^{\circ}$ associé à un fil à plomb pour la mesure de la hauteur solaire. Une boussole de randonnée de sensibilité $1^{\circ}$ mesure l'azimut. La visée et la recherche de l'ombre minimale de la boîte fournissent la mesure des paramètres étudiés.

En marge des travaux entrepris, le théodolite a servi de support d'expérimentation pour l'initiation à l'astronomie que ce soit pour des scolaires ou des adultes [3]. Son usage s'impose pour la détermination du flux d'ensoleillement maximum d'un panneau solaire photovoltaïque.

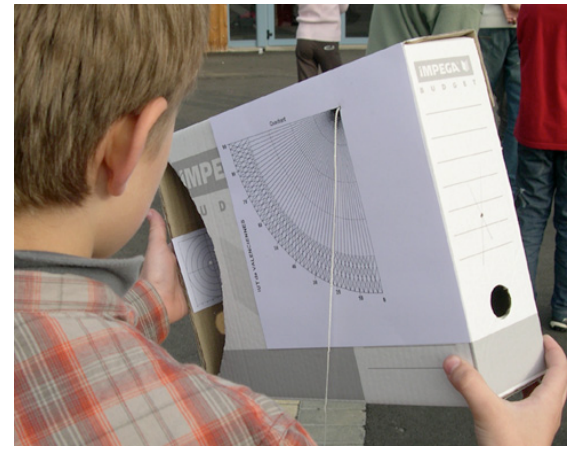

fig 1 : Mesures de l'élévation locale du soleil

\subsection{Le logiciel d'animation de la course du soleil}

L'étude se limite ici au mouvement apparent du Soleil dans le ciel vu d'un point de la terre. L'essentiel de la programmation a porté sur la création des différents objets $2 \mathrm{D}$ et $3 \mathrm{D}$ nécessaires à la constitution d'une sphère armillaire [4]. L'implémentation s'est faite sous le logiciel Matlab®, d'une part pour son jeu d'instructions propres à la représentation $2 \mathrm{D}$ et $3 \mathrm{D}$ et, d'autre part, pour la connaissance et la pratique académiques qu'en ont les étudiants $[5,6]$.

Une fois la latitude d'un lieu sur la terre et la date choisis par l'utilisateur, le programme anime le mouvement de rotation apparent sur 24 heures de la voûte céleste vu depuis ce lieu. Il reproduit la luminosité jour-nuit. Il place de plus un gnomon -c'està-dire un style- au centre du plan local matérialisant ainsi le point d'observation et affiche son ombre. Une fenêtre donne les coordonnées locales du soleil.

L'extrait figé de la course du soleil à la date du 28 Octobre concerne un point de latitude $50^{\circ} 51^{\prime} \mathrm{N}$ de la région de Bruxelles (figure 2).

\subsection{Le calcul des coordonnées locales}

Cette partie traite des fonctions astronomiques [7] développées par l'équipe enseignants/étudiants sous le langage Maple ${ }^{\mathrm{TM}}$ pratiqué dans la formation.Les algorithmes sont donnés en annexe.

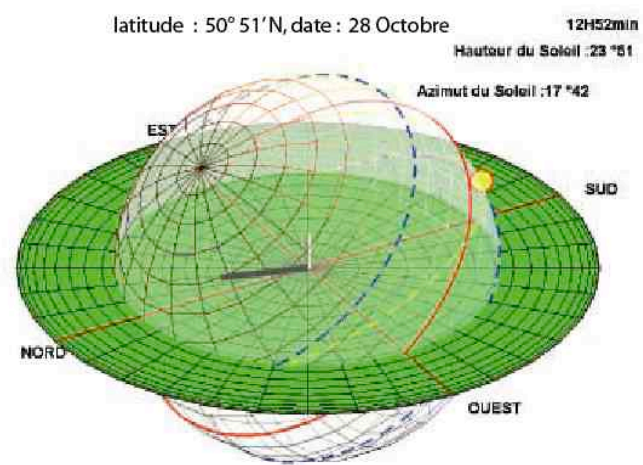

fig 2 : Extrait de l'animation de la course du soleil

Elles fournissent le passage des coordonnées horaires aux coordonnées locales. Dans la figure 3, $\omega$ est l'angle horaire, $\delta$ est la déclinaison c'est à dire l'angle entre l'équateur céleste et l'écliptique et $\Phi$ est la latitude du lieu d'observation, a est l'azimut et h la hauteur du soleil. Le changement de coordonnées est donné par les formules de Gauss $(1,2,3)$.

$\sin (h)=\sin (\Phi) \sin (\delta)+\cos (\Phi) \cos (\delta) \cos (\omega)$

$\cos (h) \sin (a)=\cos (\delta) \sin (\omega)$

$\cos (h) \cos (a)=-\sin (\delta) \cos (\Phi)+\cos (\delta) \sin (\Phi) \cos (\omega)$

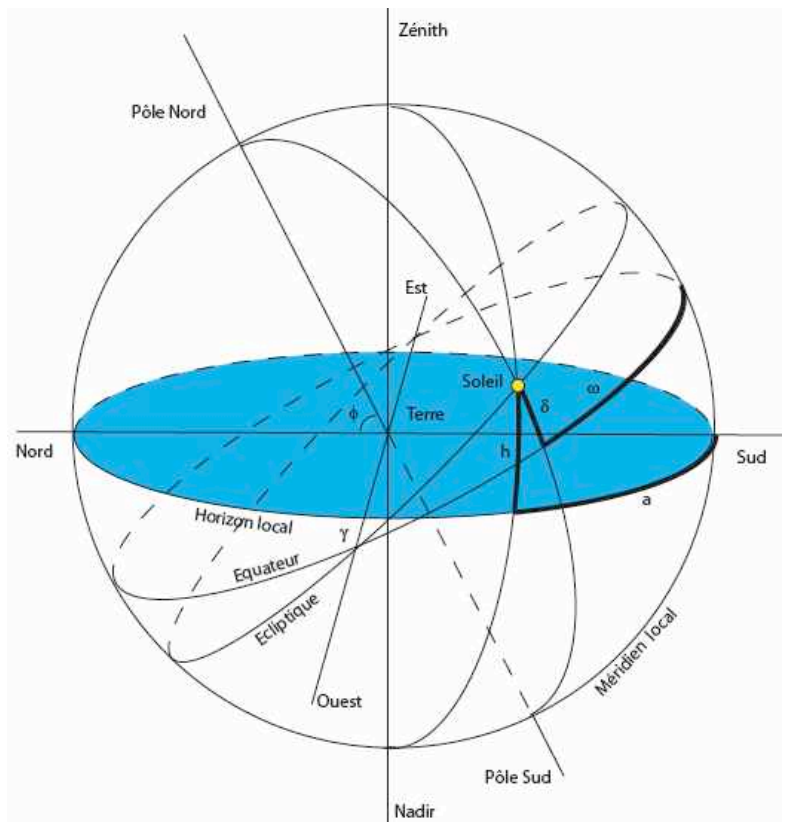

fig 3 : Coordonnées horaires et coordonnées locales

$\begin{array}{lrr}\text { La fonction } & \text { Tempsolaire- } \\ \text { Vrai(Longitude, } & \text { Tempslegal, jour, }\end{array}$


mois ) renvoie le Temps solaire vrai en heures, Longitude est la longitude du lieu d'observation, TempsLegal est l'heure donnée à la montre pour le jour et le mois de l'année courante.

La fonction soleillocal (latitude, longitude, TempsLegal, jour, mois) renvoie une liste des 2 coordonnées locales en degrés du soleil, le premier élément est la hauteur du soleil sur l'horizon, le second est l'azimut du soleil sur l'horizon local par rapport au méridien local.

Les fonctions ont été validées par un calcul d'éphémérides locales proposé dans le diagramme solaire de la figure 4. Sur celui-ci, les rayons gradués représentent l'azimut, les cercles concentriques la hauteur du soleil. Le trait noir gras représente ces deux valeurs pour la journée du 21 Mars. Les nombres placés à proximité indiquent l'heure légale.

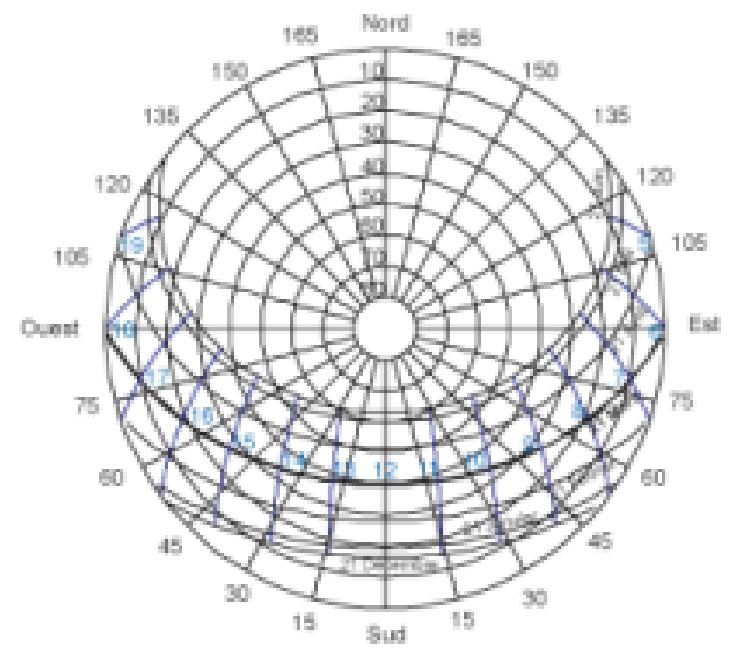

Course du Soleil Bnweles $00751 \mathrm{~N}$

\section{fig 4 : Diagramme solaire}

Les éphémérides offrent ainsi une alternative au logiciel d'animation ci-dessus pour le pilotage en position du système autonome d'orientation de panneaux photovoltaïques. Un tel choix sera dicté par la quantité d'informations à stocker dans l'automatisme.

\section{LE DISPOSITIF EXPÉRIMENTAL}

La question est ici de déterminer le gain apporté par la solution du panneau orientable par rapport au panneau fixe.

Pour cela les étudiants ont conçu, réalisé et utilisé un instrument de mesures de performances de cellules photovoltaïques.

Le dispositif se compose d'un support et d'un caisson articulé sur une platine munie d'un axe dont le plan mécanique est donné en annexe.
Le caisson contient 9 cellules photovoltaïques et la connectique afférente. Deux borniers y sont fixés pour les mesures de tension et d'intensité. Un rapporteur est également installé dans un angle supérieur de la caisse afin de pouvoir orienter le dispositif selon l'élévation choisie. La boussole posée sur la platine détermine l'azimut. (Figure 5).

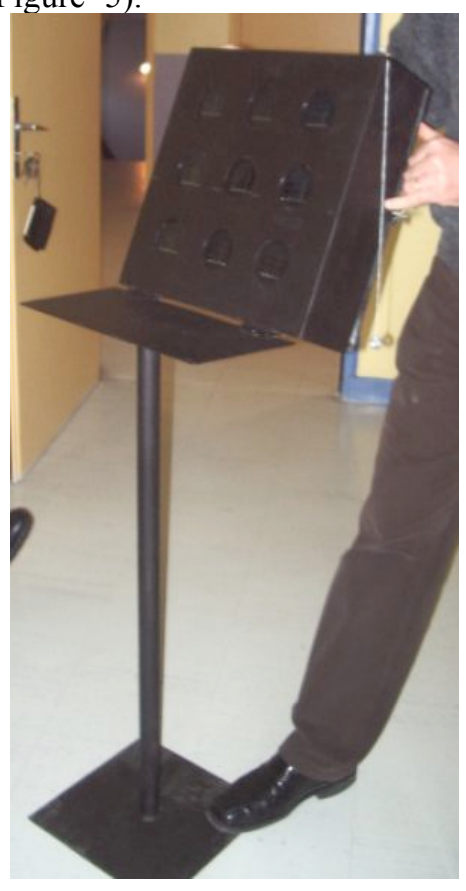

fig 5 : Dispositif de mesures

Les cellules solaires en silicium amorphe issues de lampes de jardin sont connectées suivant une structure série-parallèle (Figure 6).

La charge est une association série de 3 piles rechargeables de 1,2V chacune. Chaque groupe de cellule est protégé par une diode anti-retour. Les cellules sont assemblées sur un support mécanique à deux degrés de liberté. Le support est adaptable à d'autres types de cellules photovoltaïques comme les cellules en silicium poly-cristallin et monocristallin et cellules CIS, CGS, CGIS,CdTe voire des films minces à effet photovoltaïques mais il devrait pouvoir être motorisé pour d'autres applications [8].

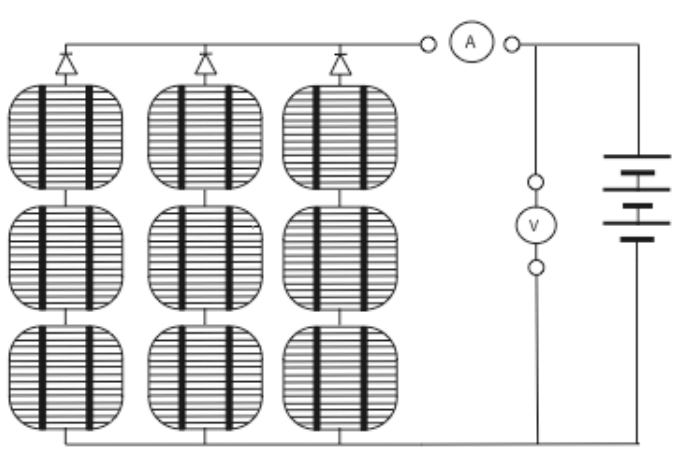

fig 6 : Schéma de branchement des cellules photovoltaïques 
La campagne de mesures des performances consiste à déterminer la puissance maximale du module selon son orientation par rapport au soleil à différentes dates et heures. Le panneau est positionné successivement à $l^{\prime}$ horizontal, plein Sud avec une hauteur de $60^{\circ}$ puis dans le flux solaire maximum grâce aux outils de la section précédente. Les calculs des puissances photoélectriques ont été traités durant une semaine. Elles concernent le point du globe de latitude $50^{\circ} 10^{\prime} 20^{\prime}$ " Nord et de longitude $3^{\circ} 14^{\prime} 08^{\prime}$ " Est situé dans la région de Cambrai (Nord). Cependant, seuls les résultats d'une journée sont proposés en figure 7 .

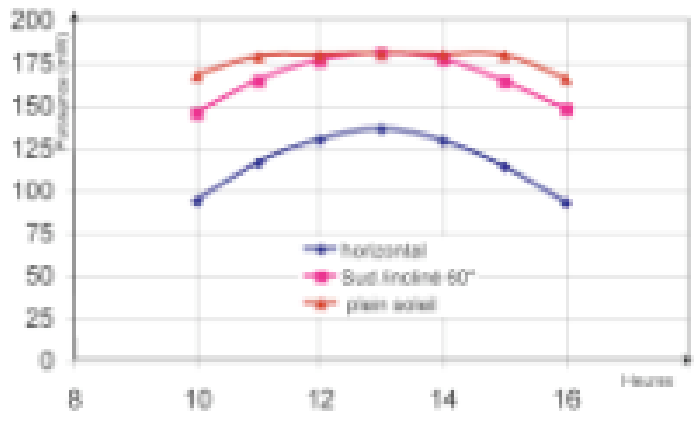

fig 7. Puissance photovoltaïque de cellules en silicium amorphe pour la journée du 12 Février 2008

\section{CONCLUSION ET PERSPECTIVES}

L'article a présenté une réalisation basée sur le thème des énergies renouvelables et plus particulièrement de l'énergie solaire sous sa nature photovoltaïque. S'inscrivant dans la démarche de la pédagogie par projet, il a permis la collaboration d'étudiants de différents cursus et d'enseignants de disciplines scientifiques et techniques. Par ailleurs, la méthode de gestion de projet PDCA -Plan Do Check Act- a conduit les travaux de la conception jusqu'à leur réalisation [9]. C'est la mise en valeur des travaux d'étudiants lors de manifestations comme la Fête de la Science ou la Journée Portes Ouvertes qui créent ainsi la synergie entre étudiants et enseignants. Elle suscite par effet d'osmose de nouvelles motivations et laisse ainsi augurer de nouvelles perspectives. L'usage de lampes spectrales pour un travail sur différentes longueurs d'onde d'une part et l'utilisation de cellules à spectre solaire différent d'autre part, la connexion avec un électrolyseur, la réalisation d'une carte d'acquisition à micro-contrôleur, la motorisation du support constituent quelques exemples. Dans le cadre d'une coopération internationale, le dispositif fera l'objet d'un mémoire de fin d'études de licence professionnelle sur le thème de la télémesure appliquée au domaine du solaire.

Hormis la partie mécanique, un avantage non négligeable de la démarche est de pouvoir reproduire à moindre coût ces expériences qui gravitent autour des sciences comme l'astronomie, l'électronique et les mathématiques appliquées. Tous les modules décrits seront nécessaires à la phase suivante de définition et de réalisation d'un asservissement en position d'un panneau solaire à partir du modèle géocentrique programmé. Adapté à l'alimentation d'un électrolyseur, un tel équipement offrira ainsi la découverte d'un système énergétique global [10].

\section{Remerciements}

L'Institut Universitaire de Technologie de Valenciennes a mis à la disposition du groupe d'étudiants et d'enseignants une salle équipée d'ordinateurs et de logiciels adéquats, qu'il en soit ici vivement remercié. Tous ces savoir-faire pluridisciplinaires ont fait l'objet d'exposés dans le cadre de mobilité Socrates.

\section{Bibliographie}

[1] Bécar J.P., Robert F., Canonne J.C., Vermeiren L. Exemples de synergies entre les mathématiques appliquées et les sciences et techniques, Actes du colloque CETSIS 07 Bordeaux, 29-31 Octobre 2007.

[2] http://www.imcce.fr

[3] Robert F., Bécar J.P., Canonne J.C., Vermeiren L, La course du soleil et applications, Exposé dans le cadre Université pour tous, Valenciennes Les Tertiales, 31 Janvier 2008

[4] Dutarte P. , Les Instruments de l'Astronomie Ancienne, Paris, Editions Vuibert, 2006.

[5] Robert F., Bécar J.P., Canonne J.C., Un exemple de projet pluridisciplinaire : Modélisation dynamique et mesures de la course du soleil, Actes du colloque CNRIUT 07, Thionville, 31 Mai-2 Juin 07

[6] Bécar J.P., Canonne J.C., Robert F.,Vermeiren L., An example of project based learning: modelling and measurements of the sun trajectory, Proceedings of IADAT07, Palma de Mallorca, 7-9 Juillet 2007.

[7] Meeus J., Astronomical Algorithms. William-Bell Inc., 1991

[8] Bécar J-P., Robert F., Vermeiren F., Canonne J-C., Multidisciplinary teachings applications on the subject : photovoltaic solar energy, IADAT-ice2008 International Conference on Energy, Bilbao (Spain), June 26-28, 2008

[9] Bécar J.-P., « Teachings with projects : sun tracking measurements», conference as invited speaker, Satakunta Polytechnic Pori Finland, April 2007.

[10] Bernard J., Bécar J.P., Vermeiren V., Maquette pédagogique de pile à combustible, Actes du 14 ème colloque CNRIUT 08, Lyon, 29-30 Mai 2008

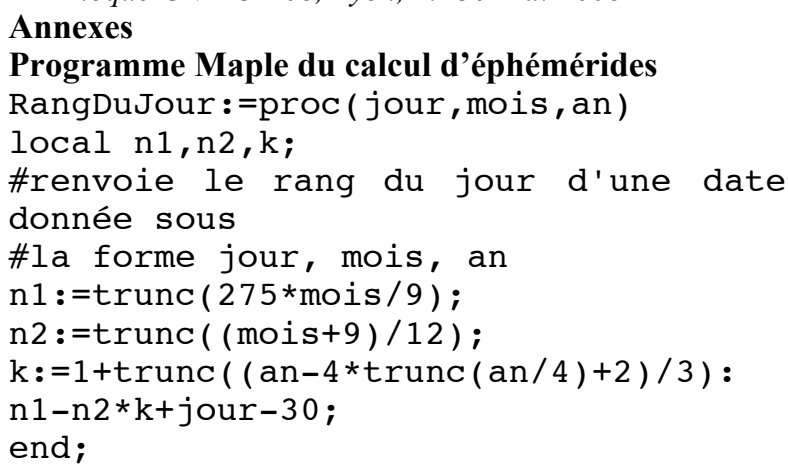

EquationDuTemps: $=$ proc $(\mathrm{n})$ 


\author{
\#Renvoie l'écart en heures entre le \\ temps solaire vrai et le temps so- \\ laire moyen \\ \#n est le Rang du jour dans l'année \\ local beta,d2r,r2d, et; \\ \#beta est l'angle horaire du soleil \\ en degrés \\ beta: $=360 * \mathrm{n} / 366$; \\ d2r:=Pi/180; \#facteur de conversion \\ degres vers radians \\ r2d:=180/Pi;\#facteur de conversion \\ radians vers degres \\ et $:=-0.0002+0.4197 * \cos ($ beta $* d 2 r)-$ \\ $7.3509 * \sin ($ beta*d2r) \\ $-3.2265 * \cos (2 *$ beta*d2r) \\ $-9.3912 * \sin (2 *$ bet $a * d 2 r)-$ \\ $0.0903 * \cos (3 *$ beta*d2r) - \\ $0.3361 * \sin (3 *$ beta*d2r): \\ evalf (et/60); \\ end;
}

TempsSolaireVrai := proc(Longitude, TempsLegal, jour, mois)

local fuseau, n, EteHiver; fuseau := 1 ;

$\mathrm{n}:=$ jour $+31 *$ mois $-31 ;$

if mois $<3$ then $n$ else $n:=n-$

trunc $(0.4 *$ mois +2.3$)$ end if;

EteHiver $:=0$;

if $89<=\mathrm{n}$ and $\mathrm{n}<=299$ then Ete-

Hiver := 1 end if;

TempsLegal - fuseau - EteHiver -

1/15*Longitude + EquationDuTemps (n); end proc;

Soleillocal := proc(latitude, longitude, Tempslegal, jour, mois)

local $\mathrm{n}, \mathrm{d} 2 \mathrm{r}, \mathrm{r} 2 \mathrm{~d}$, delta, omega, hauteur, azimut, tsv, sazimut;

$\mathrm{d} 2 \mathrm{r}:=1 / 180 * \mathrm{Pi}$;

$\mathrm{r} 2 \mathrm{~d}:=(180) /(\mathrm{Pi})$;

$\mathrm{n}:=$ jour $+31 *$ mois -31 ;

if mois < 3 then $n$ else $n:=n-$

trunc $(0.4 *$ mois +2.3$)$ end if;

delta :=

evalf $(23.45 * \sin (72 / 73 * d 2 r *(n-81)))$; tsv := TempsSolaireVrai (longitude,

Tempslegal, jour, mois);

omega $:=15 * t s v-180$;

hauteur := arc-

$\sin (\cos (\operatorname{delta} a d 2 r) * \cos ($ omega $* d 2 r) * \cos$ (lati-

tude*d2r) $+\sin ($ delta*d2r)*sin (latitude $* d 2 r)) * r 2 d$;

sazi-

mut: $=$ evalf $((\cos ($ delta*d2r)*sin (omega* $\mathrm{d} 2 \mathrm{r})) /(\cos ($ hauteur*d2r)) );

azimut := ar -

$\cos ((\cos ($ delta $* d 2 r) * \cos ($ omega $* d 2 r) * s i$ $\mathrm{n}($ latitude*d2r) sin (delta*d2r)* cos ( lat itude*d2r) )/( $\cos ($ hauteur*d2r) ))*r2d; if sazimut $<0$ then azimut $:=360-$ azimut end if;

[evalf(hauteur), evalf(azimut)] ;

end proc;

Plan du support des cellules photovoltaïques

Le support se compose de deux parties. Un pied construit en tube d'acier fixé sur un socle (figure 8) constitue l'axe de rotation pour le positionnement des azimuts. La figure 9 représente un caisson entièrement fabriqué en tôle d'aluminium de 3 millimètres d'épaisseur. Fixé sur une platine articulée, il peut s'orienter selon les différentes élévations. Il repose sur l'axe de rotation précédent, contient les cellules photovoltaïques et la connectique appropriée.
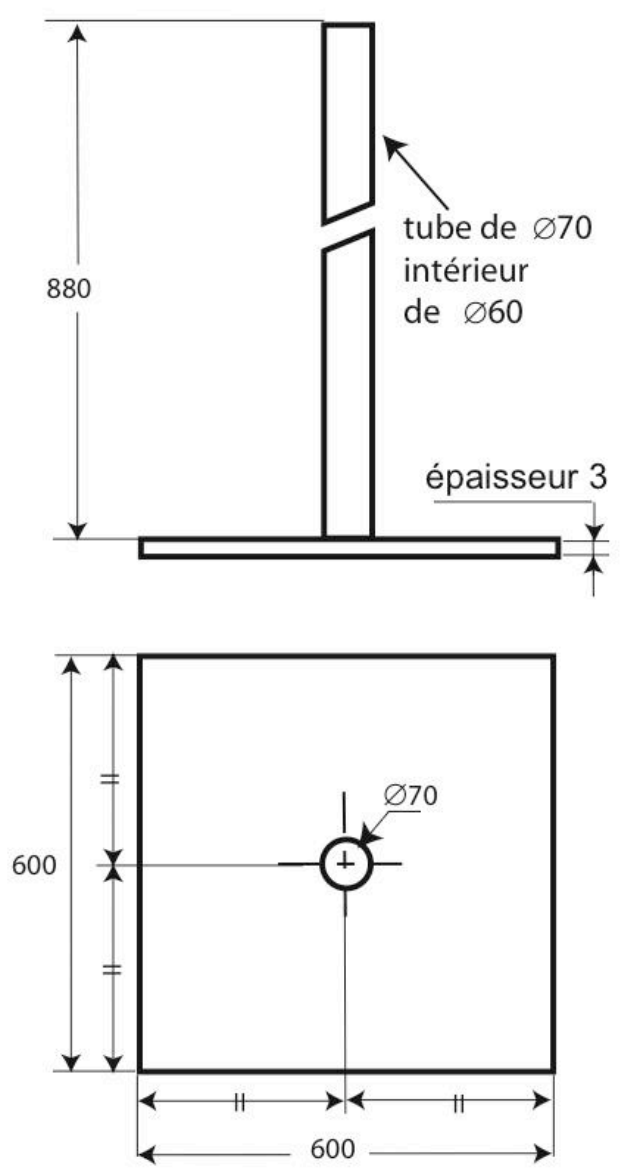

fig 8: Plan du pied support du caisson 


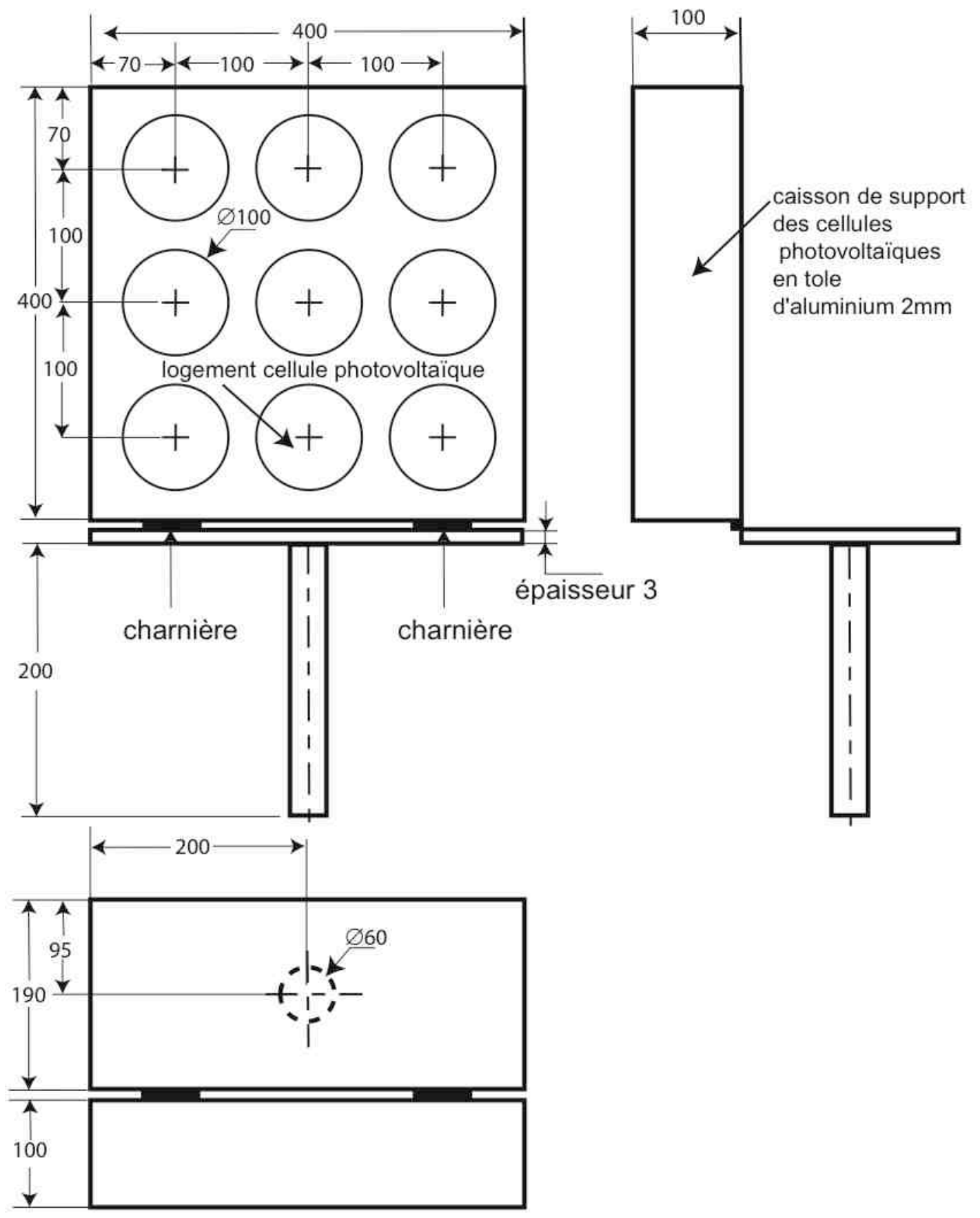

fig 9. Caisson pour cellules photovoltaïques 